\title{
Crossover Frequency and Transmission-Line Matrix Formalism of Electromagnetic Shielding Properties of Laminated Conductive Sheets
}

\author{
S. M. Benhamou ${ }^{1,2 *}$, M. Hamouni ${ }^{2}$ and F. Ould-Kaddour ${ }^{3}$ \\ ${ }^{1,2}$ Ecole Supérieure en Sciences Appliquées de Tlemcen, BP 165 RP Bel horizon ,13000 Tlemcen, Algerie. \\ ${ }^{2}$ Laboratoire de recherche sur les macromolécules (LRM), département de physique, Faculté des sciences, \\ Université de Tlemcen, BP 119, Tlemcen, 13000, Algérie. \\ ${ }^{3}$ Laboratoire de physique théorique, département de physique, Faculté des sciences, Université de Tlemcen, BP \\ 119, Tlemcen, 13000, Algérie. \\ *Corresponding author, E-mail: sm.benhamou@yahoo.com
}

\begin{abstract}
This paper proposes an approach to calculate the crossover frequency of each layer in the multilayered shield and subsequently that of structure constructed by $\mathrm{n}$ layers. This important frequency provides a useful approximation for field penetration in a conductor. It is used in a wide variety of calculations. It is in this context that a simplification of the transmission-line matrix formalism for laminated conductive sheets is done using this frequency. Two ranges of frequency are considered: lower and higher than the crossover frequency. Simples formulas and easy to use of the reflection loss, the internal reflection, the absorption loss and the electromagnetic shielding effectiveness of laminated shield are obtained. Analysis is carried out for the study of two shields: i) single shield of carbon nanotube polymer composites (CNTs), ii) multilayered shield constructed with Nickel-carbon nanotube polymer composites-Aluminum (Ni-CNTs-Al).
\end{abstract}

Keywords: Crossover frequency, Transmission-line matrix formalism, Shielding effectiveness.

\section{Introduction}

Multiple or laminated shields are obtained by a stratification of two or more sheets of different materials. They are used because of the advantages of shielding performance or for other technical reasons occurring. The transmission-line matrix formalism is used for determination of electromagnetic shielding properties of multilayered mediums. We consider a structure of $n$ layers, with constitutive parameters: the impedance $Z_{j}$ and the thickness $d_{j}$, as showed in figure 1 .

For each layer, we associate a transfer matrix defined as [1]:

$$
M_{j}=\left[\begin{array}{cc}
\cosh \left((1-\mathrm{i}) \frac{d_{j}}{\delta_{j}}\right) & -Z_{j} \sinh \left((1-\mathrm{i}) \frac{d_{j}}{\delta_{j}}\right) \\
-\frac{1}{Z_{j}} \sinh \left((1-\mathrm{i}) \frac{d_{j}}{\delta_{j}}\right) & \cosh \left((1-\mathrm{i}) \frac{d_{j}}{\delta_{j}}\right)
\end{array}\right]
$$

where $\delta_{j}$ is a skin depth of layer $j$, the value for which the fields reduce its value to $36.8 \%$ of the maximum. The intrinsic impedance of the $j^{\text {th }}$ layer for a good conductor is given by [2]:

$$
Z_{j}=(1+\mathrm{i}) / \sigma_{j} \delta_{j}
$$

where $\sigma_{j}$ is the electrical conductivity.

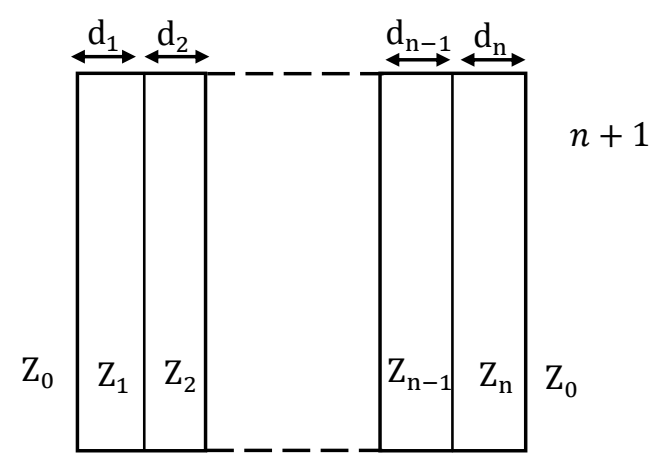

Fig. 1. Scheme of the multilayered shield.

The characteristic matrix of the whole structure is given by the product of the different characteristic matrix of each layer [3]:

$$
M=\prod_{j=1}^{n} M_{j}=\left[\begin{array}{ll}
M_{11} & M_{12} \\
M_{21} & M_{22}
\end{array}\right]
$$

According to Naishadham [4], we are in the position to state the coefficient of transmission $T$ for the $j$ layer as: 


$$
T=\frac{2\left[M_{22}\left(M_{11} Z_{j-1}-M_{12}\right)+M_{12}\left(M_{22}-Z_{j-1} M_{21}\right)\right]}{\left(M_{11} Z_{j-1}-M_{12}\right)+Z_{j+1}\left(M_{22}-Z_{j-1} M_{21}\right)}
$$

The electromagnetic shielding effectiveness SE of a material is defined by:

$$
S E(d B)=-20 \log |T|
$$

The shielding effectiveness given in (5) can be broken into the sum of three terms each representing one of the phenomena of reflection loss, absorption loss, and multiple reflections. These factors add to give [5]:

$$
S E(d B)=R(d B)+A(d B)+B(d B)
$$

where $R(d B)$ represents the reflection loss caused by reflection at the left and right interfaces, it increases with increasing permittivity and conductivity of shield, $A(d B)$ represents the absorption loss of the wave as it proceeds through the barrier, it necessitates electric and/or magnetic dipoles in the shield [6] and $B(d B)$ represents the additional effects of multiple re-reflections and transmissions inside the materials [7].

The calculation of the EMI shielding of plane electromagnetic radiation by a multilayered shield using the matrix formalism is tedious; in addition, the expressions resulting from this approach are cumbersome. This formalism can be used for all samples that fulfill the classical good conductor approximation for both electrically thin (thickness < skin depth) as well as electrically thick (thickness $>$ skin depth) over the frequency range of interest. This approximation has been used by Colaneri and Shaklette for composite material effectiveness shielding [8]. That is how is we require to calculate the skin depth and therefore the crossover frequency, at which $d_{j}=\delta_{j}$, of each layer in the multilayered shield and subsequently that of structure constructed by $n$ layers.
In this paper, after introduced the reasoning leading to the crossover frequency expression of any layer in a multilayer structure and that of the whole layers, we propose simplified formulas of reflection loss, multiple re-reflections, absorption loss, and shielding effectiveness of multilayered electromagnetic shielding.

The simplification is carried out in two steps: the first is the classical good conductor approximation, by assuming that $\sigma / \omega \varepsilon$ ( $\omega$ and $\varepsilon$ are, respectively, the pulsation and the permeability). The second step is based on considering the two frequency ranges: first, when the frequency is much lower than the crossover frequency, the second, in the higher frequency range than the crossover frequency. Analysis is carried out for the study of these phenomena for single (CNTs) and laminated shields (Nickel - (CNTs) - Aluminum) as a function of frequency.

\section{Crossover Frequency of Multilayered Shield}

The simplification procedure of the matrix formalism is based on knowing the crossover frequency of laminated structure. For this purpose we will first of all address the reasoning that has led us to calculate this frequency and its properties.

In order to make a correct calculation of the skin depth and therefore the crossover frequency of each layer in the laminated sheet, the multilayer shield of figure 1 has been disjoined. This can easily be shown graphically, as illustrated in figure 2. Just at or below the surface of the first sheet, the amplitude of the electric field incident is given as $E_{x 0}$. We assume an air interface with the multilayered structure.
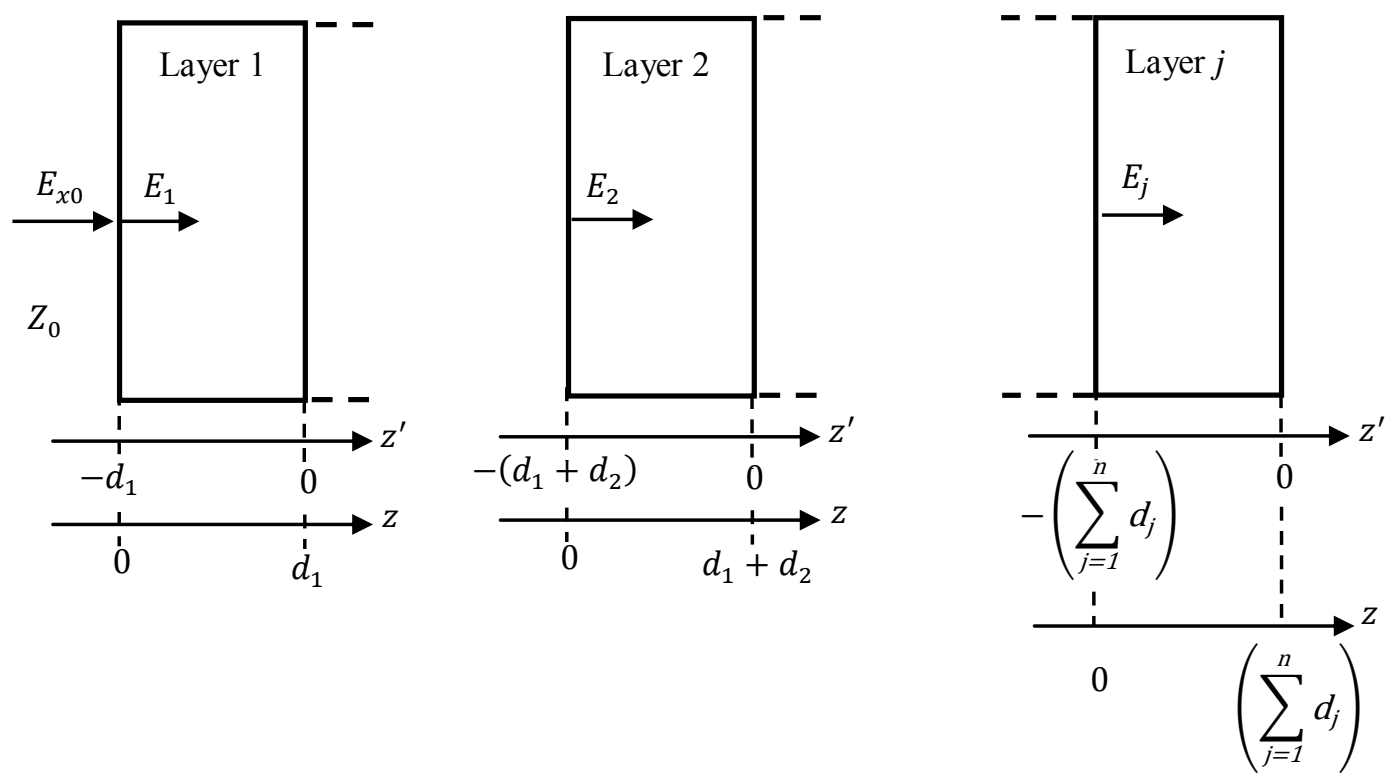

Fig. 2. Propagation field into the laminated slab. 
Notice that a temporary z' coordinate system is used to simplify the derivation. Referring to figure 2 , at any point on the line, the field is given by:

$$
E_{x}\left(z^{\prime}\right)=E_{0} e^{-k_{c} z^{\prime}}
$$

where $E_{0}$ is the amplitude of the incident wave and $k_{c}$ is the attenuation constant determines the penetration of the wave into a medium, given by:

$$
k_{c}=\sqrt{\pi f \mu \sigma}
$$

where $f$ is the frequency of electromagnetic wave.

For the first layer, the relation (7) is written as;

$$
E_{x}\left(z^{\prime}\right)=E_{1} e^{-k_{c 1} z^{\prime}}
$$

Using (9) at $z^{\prime}=-d_{1}$, we can have $E_{1}$ in the terms of the field at the surface $E_{x 0}$, and therefore;

$$
E_{x}\left(z^{\prime}\right)=\frac{e^{-k_{c 1} z^{\prime}}}{e^{+k_{c 1} d_{1}}} E_{x 0}
$$

Shifting the coordinate system as indicated in the figure 2 give us:

$$
E_{x}(z)=\frac{e^{-k_{c 1}\left(z-d_{1}\right)}}{e^{+k_{c 1} d_{1}}} E_{x 0}
$$

The skin depth $\delta$ is a measure of the depth to which the radiation will penetrate within the material with a decrease in intensity to $1 / e$ of its original strength [8]. This intensity decrease is shown in figure 3 .

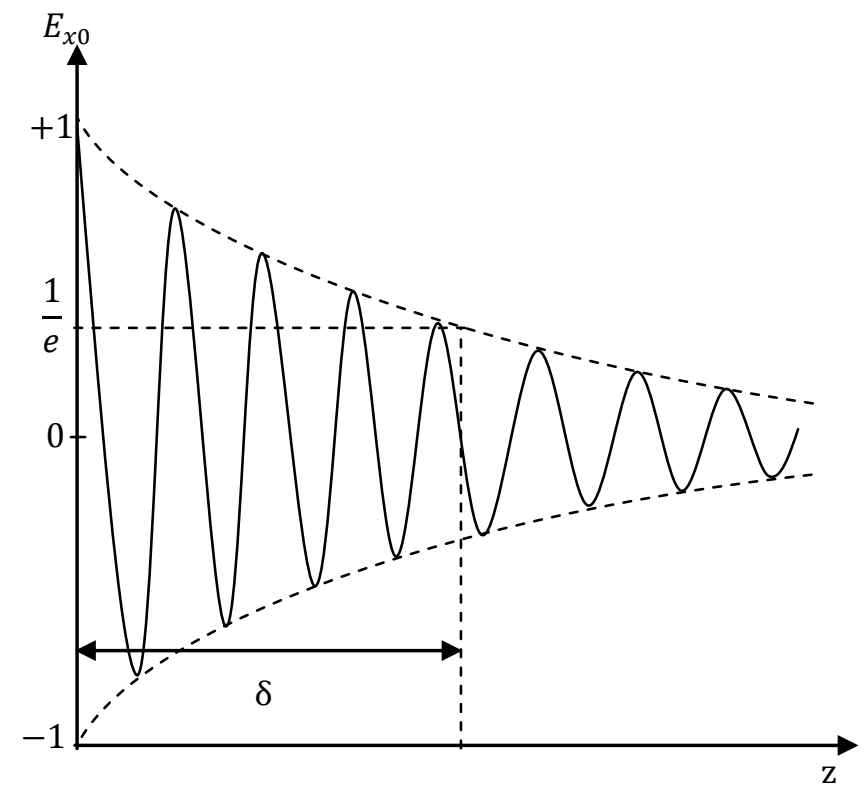

Fig. 3. Lowering of electromagnetic wave intensity in conductive medium.

$$
\begin{gathered}
\frac{E_{x}(z)}{E_{x 0}}=\frac{e^{-k_{c 1}\left(\delta_{1}-d_{1}\right)}}{e^{+k_{c 1} d_{1}}}=\frac{1}{e} \\
\delta_{1}=\frac{1}{k_{c 1}}=\sqrt{1 /\left(\sigma_{1} \mu_{1} \pi f\right)}
\end{gathered}
$$

The field at any point of the second layer is given similarly by:

$$
E_{x}(z)=E_{2} e^{-k_{c 2}\left(z-d_{1}-d_{2}\right)}
$$

For the tangential field is equal at the surface $\left(z=d_{1}\right), E_{2}$ is expressed by:

$$
E_{2}=\frac{1}{e^{+k_{c 1} d_{1}} e^{+k_{c 2} d_{2}}} E_{x 0}
$$

Therefore, the equation (14) is written as:

$$
E_{x}(z)=\frac{E_{2} e^{-k_{c 2}\left(z-d_{1}-d_{2}\right)}}{e^{+k_{c 1} d_{1} e^{+k_{c 2} d_{2}}}} E_{x 0}
$$

As the skin depth $\delta_{2}$ corresponds to decrease in intensity to $1 / e$, we can write:

$$
\frac{E_{x}(z)}{E_{x 0}}=\frac{E_{2} e^{-k_{c 2}\left(\delta_{2}-d_{1}-d_{2}\right)}}{e^{+k_{c 1} d_{1}} e^{+k_{c 2} d_{2}}}=\frac{1}{e}
$$

Thus $\delta_{2}$ is given by:

$$
\delta_{2}=\frac{d_{1}\left(k_{c 2}-k_{c 1}\right)+1}{k_{c 2}}
$$

Similar to treatment in the second layer, the third skin depth layer is expressed by:

$$
\delta_{3}=\frac{d_{1}\left(k_{c 3}-k_{c 1}\right)+d_{2}\left(k_{c 3}-k_{c 2}\right)+1}{k_{c 3}}
$$

The general formula of skin depth of each conductor is summarized as:

$$
\delta_{j}=\frac{d_{1}\left(k_{c j}-k_{c 1}\right)+d_{2}\left(k_{c j}-k_{c 2}\right)+\cdots+d_{j-1}\left(k_{c j}-k_{c(j-1)}\right)+1}{k_{c j}}
$$

So the skin depth of $\mathrm{n}$ layers can be calculated by:

$$
\delta=\sum_{j=1}^{n} \delta_{j}=\sum_{j=1}^{n-1} \frac{d_{j}\left(k_{n}-k_{j}\right)+1}{k_{j}}
$$

The crossover frequency is the frequency which ensures equality between the skin depth and the layer thickness [8]. Hence, when doing this, we can establish the crossover frequency expression of each layer as follows:

first layer:

second layer:

$$
f_{c 1}=\frac{1}{\pi d_{1}^{2} \sigma_{1} \mu_{1}}
$$

$$
f_{c 2}=\frac{1}{\pi\left[\left(d_{2}-d_{1}\right) \sqrt{\sigma_{2} \mu_{2}}+d_{1} \sqrt{\sigma_{1} \mu_{1}}\right]^{2}}
$$

third layer:

$$
f_{c 3}=\frac{1}{\pi\left[\left(d_{3}-d_{2}-d_{1}\right) \sqrt{\sigma_{3} \mu_{3}}+d_{2} \sqrt{\sigma_{2} \mu_{2}}+d_{1} \sqrt{\sigma_{1} \mu_{1}}\right]^{2}}
$$

$\mathrm{j}^{\text {th }}$ layer:

$$
f_{c j}=\frac{1}{\pi\left[\left(d_{j}-\sum_{j=1}^{n-1} d_{j}\right) \sqrt{\sigma_{j} \mu_{j}}+\sum_{j=1}^{n-1} d_{j} \sqrt{\sigma_{j} \mu_{j}}\right]^{2}}
$$


Lastly, the crossover frequency of the whole structure, where we have equality between the skin depth and the thickness of laminated shield, can be calculated by;

$$
f_{c}=\frac{1}{\pi\left[\sqrt{\sigma_{n} \mu_{n}} \sum_{j=1}^{n} d_{j}-\sum_{j=1}^{n-1} d_{j}\left(\sqrt{\sigma_{n} \mu_{n}}-\sqrt{\sigma_{j} \mu_{j}}\right)\right]^{2}}
$$

\section{Transmission-line Matrix formalism for conductive sheets}

We first consider a single layer then proceed to multilayered shield.

\subsection{Single shield}

The characteristic matrix $M_{j}$ of conductive sheet, $Z_{j} \ll Z_{0}$, in contact with two semi-infinite air media, so that $Z_{j-1}=$ $Z_{j+1}=Z_{0}=377 \Omega$ reduces from (1) to:

$$
M_{j}=\left[\begin{array}{cc}
1 & 0 \\
-\frac{\tanh \left((1-\mathrm{i}) \frac{d_{j}}{\delta_{j}}\right)}{Z_{j}} & 1
\end{array}\right] \cosh \left((1-\mathrm{i}) \frac{d_{j}}{\delta_{j}}\right)
$$

Expression of $M_{j}$ has two limits of interest which can be taken as acceptable approximations, depending on whether the frequency is higher or lower than that at which the thickness $d_{j}$ equals the skin depth $\delta_{j}$.

- If the frequency is much lower than $f_{c j}, M_{j}$ reduces to:

$$
M_{j}=\left[\begin{array}{cc}
1 & 0 \\
-\frac{(1-i) \frac{d_{j}}{\delta_{j}}}{Z_{j}} & 1
\end{array}\right] \exp \left(\frac{d_{j}}{\delta_{j}}\right)
$$

The coefficient of transmission can therefore be written as:

$$
\frac{1}{T}=\left[1+\frac{Z_{0}(1-i) \frac{d_{j}}{\delta_{j}}}{2 Z_{j}}\right] \exp \left(\frac{d_{j}}{\delta_{j}}\right)
$$

Using (5), the relation of $S E$ becomes:

$$
S E(d B)=\underbrace{8.66 \frac{d_{j}}{\delta_{j}}}_{A(d B)}+\underbrace{20 \log \left(1+\frac{Z_{0} \sigma_{j} d_{j}}{2}\right)}_{R(d B)+B(d B)}
$$

As the absorption loss is proportional to shield thickness and is a function of $\sigma_{j}$ and $\mu_{j}$ [9] and by identification with (6), we can say that the first term of (30) represents the absorption loss $A(d B)$ and the second is the sum of reflection loss $R(d B)$ and internal multiple reflections $B(d B)$.

- If the frequency is much higher than $f_{c j}, \mathrm{M}_{\mathrm{j}}$ reduces to:

$$
M_{j}=\left[\begin{array}{cc}
1 & 0 \\
-\frac{1}{Z_{j}} & 1
\end{array}\right] \frac{\exp \left(\frac{d_{j}}{\delta_{j}}\right)}{2}
$$

In this frequency range, the contribution of the multiple reflection-loss can usually be neglected with respect to the reflection and the absorption loss because of the high penetration loss inside the shielding material [7]. The effectiveness shielding will be a sum of the reflection and the absorption loss:

$$
S E(d B)=\underbrace{8.66 \frac{d_{j}}{\delta_{j}}}_{A(d B)}+\underbrace{20 \log \left(1+\frac{Z_{0} \sqrt{\frac{\sigma_{\mathrm{j}}}{f \mu_{\mathrm{j}}}}}{10}\right)}_{R(d B)}
$$

A simple comparison between the equations (30) and (32), allows for the relation of internal multiple reflections of $j$ conductive sheet when $f \ll f_{c j}$ :

$$
B(d B)=20 \log \left[\frac{1+\frac{z_{0} \sigma_{j} d_{j}}{2}}{1+\frac{\sqrt{\frac{\sigma_{\mathrm{j}}}{f \mu_{\mathrm{j}}}}}{10}}\right]
$$

The reflection and the absorption loss expression is the same in the two frequency range $\left(f \gg f_{c j}\right.$ and $\left.f \ll f_{c j}\right)$, it's given by:

$$
\begin{gathered}
R(d B)=20 \log \left(1+\frac{Z_{0} \sqrt{\frac{\sigma_{j}}{f \mu_{j}}}}{10}\right) \\
A(d B)=8.66 \frac{d_{j}}{\delta_{j}}
\end{gathered}
$$

\subsection{Multilayered shield}

Now, we will generalize the relation of the reflection loss, the internal multiple reflections, the absorption loss and the shielding effectiveness for $n$ conductive sheets for both frequency ranges $\left(f \gg f_{c m}\right.$ and $\left.f \ll f_{c m}\right)$. Here $f_{c m}$ represents the crossover frequency of whole structure. The characteristic matrix of multilayered structure can be expressed as:

$$
M=\left[-\sum_{j=1}^{n} \frac{1}{\tanh \left((1-\mathrm{i}) \frac{d_{j}}{\delta_{j}}\right)} Z_{j}^{0} \quad 1\right] \prod_{j=1}^{n} \cosh \left((1-\mathrm{i}) \frac{d_{j}}{\delta_{j}}\right)
$$

- In the lower frequency range than $f_{c m}, M$ becomes:

$$
M=\left[\sum_{j=1}^{n} \sigma_{j} d_{j} 1\right] \prod_{j=1}^{n} \exp \left(\frac{d_{j}}{\delta_{j}}\right)
$$

The shielding effectiveness of laminated conductive layers reduces to: 


$$
S E(d B)=\underbrace{8.66 \sum_{j=1}^{n} \frac{d_{j}}{\delta_{j}}}_{A(d B)}+\underbrace{20 \log \left(1+\frac{Z_{0}}{2} \sum_{j=1}^{\mathrm{n}} \sigma_{j} d_{j}\right)}_{R(d B)+B(d B)}
$$

- In the higher frequency range than $f_{c m}, M$ reduces to:

$$
M=\left[-\sum_{j=1}^{n} \frac{1}{Z_{j}} \quad 1\right] \prod_{j=1}^{n} \frac{\exp \left(\frac{d_{j}}{\delta_{j}}\right)}{2}
$$

In this frequency range, the shielding effectiveness, with negligible internal multiple reflections, can be written as:

$$
S E(d B)=\underbrace{8.66 \sum_{\mathrm{j}=1}^{\mathrm{n}} \frac{d_{j}}{\delta_{j}}}_{A(d B)}+\underbrace{20 \log \left(1+\frac{Z_{0}}{\left.2^{n+\frac{3}{2}} \sum_{j=1}^{\mathrm{n}} \sigma_{j} \delta_{j}\right)}\right.}_{R(d B)}
$$

where

$$
\begin{gathered}
R(d B)=20 \log \left(1+\frac{Z_{0}}{2^{n+\frac{3}{2}}} \sum_{j=1}^{n} \sigma_{j} \delta_{j}\right) \\
A(d B)=8.66 \sum_{j=1}^{n} \frac{d_{j}}{\delta_{j}}
\end{gathered}
$$

The reflection and the absorption loss evolve according to the same expression regardless of the frequency range. A simple comparison between the equations (38) and (40), allows for the internal multiple reflections of $n$ multilayered conductive shield when $f \ll f_{c m}$ :

$$
B(d B)=20 \log \left[\frac{1+\frac{z_{0}}{2} \sum_{j=1}^{n} \sigma_{j} d_{j}}{1+\frac{Z_{0}}{2^{n+\frac{3}{2}}} \sum_{j=1}^{n} \sigma_{j} \delta_{j}}\right]
$$

\section{Applications}

Compared to metals, carbon nanotube polymer composites (CNTs) possess cost, weight, design flexibility, corrosion resistivity and ease of processing advantages. The high aspect ratio and extraordinary electrical conductivity of CNTs were utilized to formulate nanocomposite materials with low electrical percolation threshold and electrical resistivity [10]. These materials are very promising for applications in electromagnetic interference shielding [11].

The effect of the frequency of the incidence wave on the reflection loss, the internal multiple reflections, the absorption and the shielding effectiveness are analyzed for multilayer's shields using expressions obtained in this work. The materials selected for shielding in combination with the conductive polymer were aluminum and nickel. The material parameters conductivity, relative permeability and thickness of the considered materials and conducting polymer are listed in the Table 1. These materials are chosen to be good conductors. The crossover frequency for single shield constructed with
CNTs is calculated by (22) and is worth $f_{c j}=1.1510^{9} \mathrm{~Hz}$ whereas that of multilayered shield composed of Nickelcarbon nanotube polymer composites-Aluminum is evaluated using (26) and is worth $f_{c m}=2.210^{3} \mathrm{~Hz}$.

\section{TABLE 1}

CONDUCTIVITY, RELATIVE PERMEABILITY AND THICKNESS OF SELECTED MATERIALS.

\begin{tabular}{cccc}
\hline \hline Material & $\sigma\left({\left.\mathrm{S} . \mathrm{m}^{-1}\right)}\right.$ & $\mu_{r}$ & $\mathrm{~d}(\mathrm{~mm})$ \\
\hline Nickel & $1,1610^{7}$ & 100 & 0.1 \\
Aluminum & $3,53810^{7}$ & 1 & 0.1 \\
CNTs (single) [12] & $2.210^{4}$ & 1 & 0.3 \\
CNTs (laminated) [12] & $2.210^{4}$ & 1 & 0.1
\end{tabular}

Figure 4 shows the reflection loss evolution of: (CNTs) and (Nickel-CNTs-Aluminum) with frequency, using, respectively, the equations (34) and (41). It shows that for both shields the reflection loss decreases dramatically as the frequency increases. This could be explained by noting that the magnitude of the intrinsic impedance of material is directly proportional to the frequency; that is, as the frequency increases the impedance of material increases, and hence reflection loss is more important at lower frequency than at higher frequency.

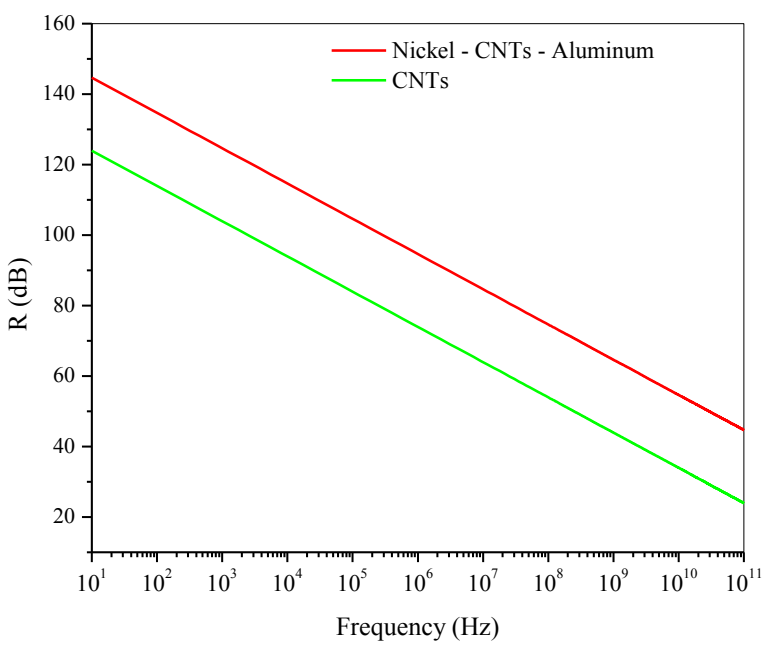

Fig. 4. Variation of the reflection loss as a function of frequency. 
For absorption loss, as shown in figure 5 plotted using (35) for single and (42) for multilayered shield; at lower than crossover frequency, it can be neglected compared with reflection and internal reflection loss. This behavior must be due to shallower skin depth of the composite with higher electrical conductivity. Above this frequency, absorption becomes dominant and increases exponentially with frequency.
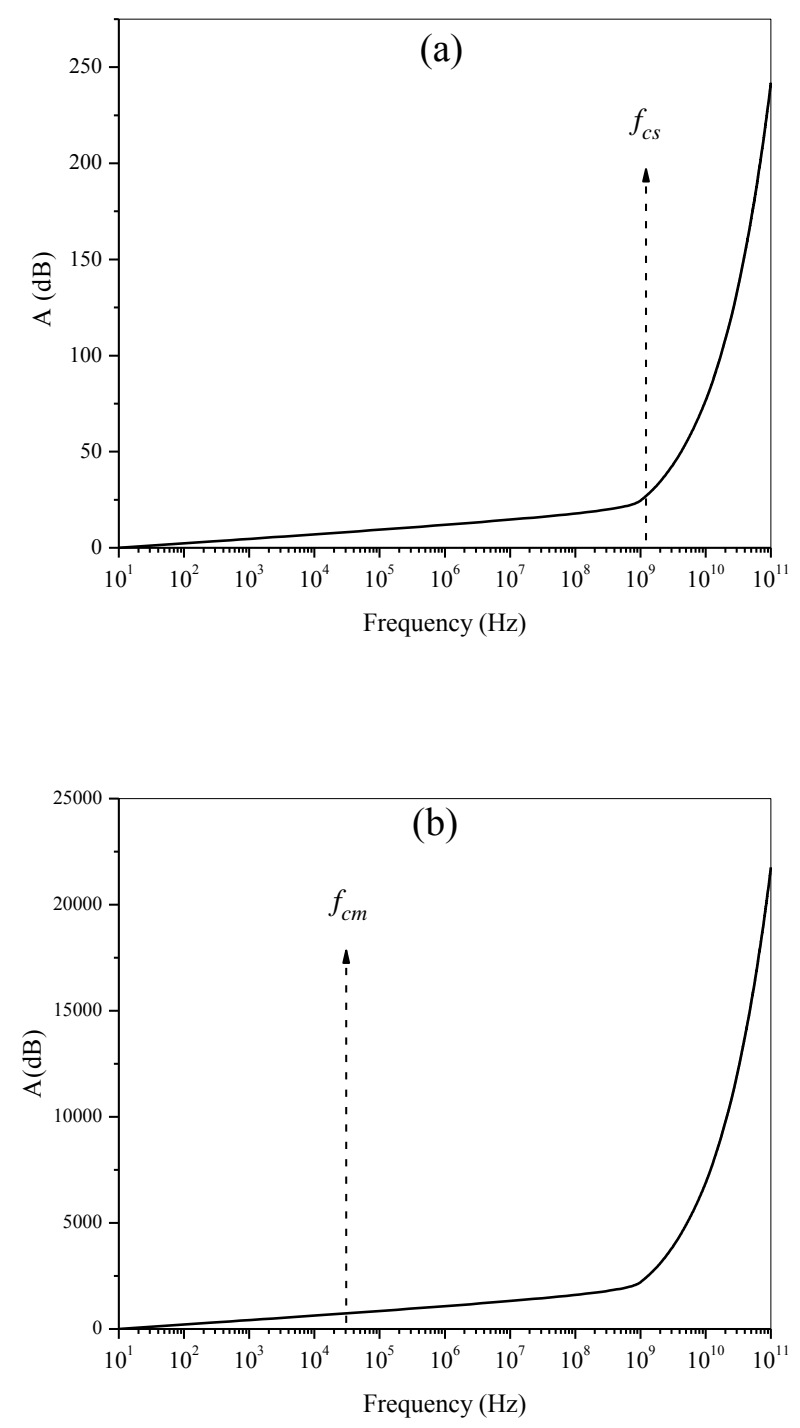

Fig. 5. Variation of the absorption loss as a function of frequency of: (a) CNTs and (b) (Nickel - CNTs - Aluminum).
Figure 6 depicts the variation of the internal reflection loss as a function of frequency, using, (33) for (CNTs) and (43) for (Nickel-CNTs-Aluminum). The internal reflection loss has a negative value and reduces the overall shielding effectiveness, which indicates that less shielding is obtained because of the multiple reflections

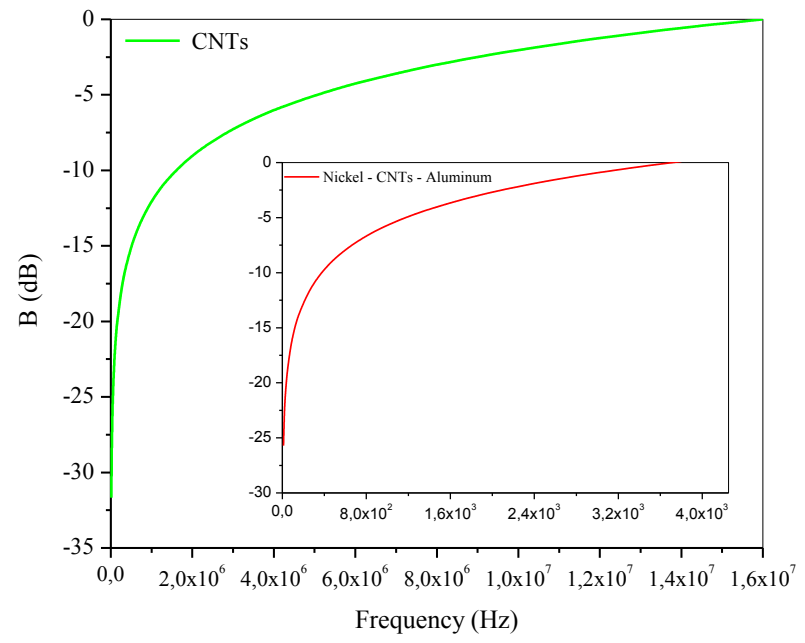

Fig. 6. Variation of the internal reflection loss as a function of frequency.

Figure 7 is plotted using (30) for single shield constructed with CNTs (when $f \ll f_{c j}$ ) and (38) for multilayered shield composed of Nickel, CNTs and Aluminum (when $f \ll f_{c m}$ ), whereas, the figure 8 is plotted using (32) for CNTs corresponding to $f \gg f_{c j}$ and (40) for (Nickel-CNTsAluminum) corresponding to $f \gg f_{c m}$; they show that the shielding effectiveness is frequency independent up to some limiting frequency (crossover frequency) after which it increases with increasing frequency. 

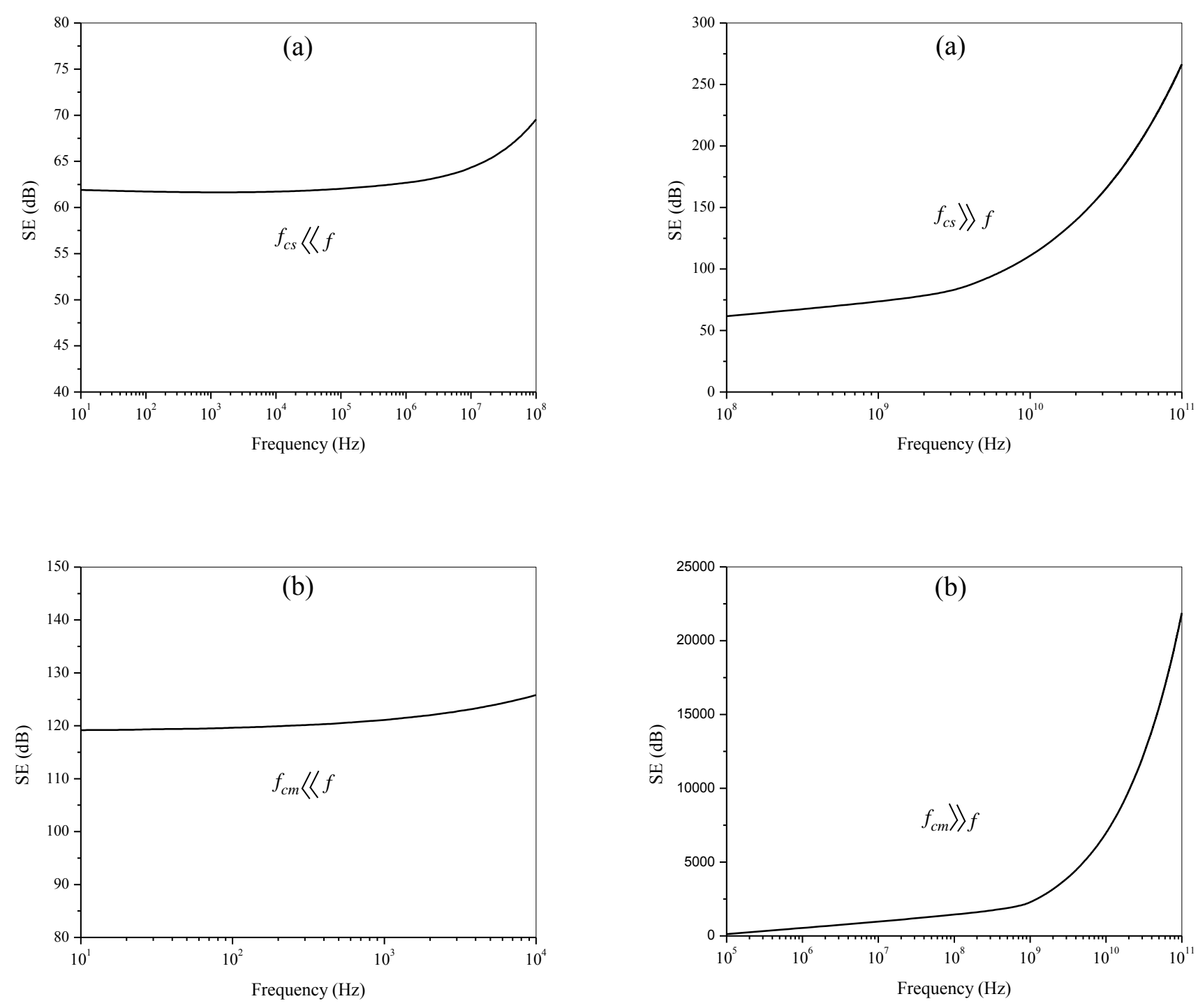

Fig. 7. Dependence of the shielding effectiveness of: (a) CNTs and (b) (Nickel-CNTs-Aluminum).

Fig. 8. Dependence of the shielding effectiveness of: (a) CNTs and (b) (Nickel-CNTs-Aluminum).

\section{Conclusions}

In this paper, a reasoning have been proposed to calculate the skin depth and therefore the crossover frequency of laminated structure. A simplification of the reflection loss, multiple rereflections, absorption loss, and shielding effectiveness formulas of multilayered conductive sheets is presented by dividing the area of frequency in two ranges: the lower and the higher frequency range than crossover frequency $f_{\mathrm{c}}$. With its easiness of using and reduced calculations time, this approximation is more interesting than the exact formulations. The reflection and the absorption loss evolve according to the same expression regardless of the frequency range. The internal reflection first increases rapidly with frequency than show a slower increase. The absorption loss first shows a negligible increase then follows a rapid increase. As summary, 
the shielding effectiveness is nearly constant at lower frequency then shows a rapid increase.

\section{References}

[1] S. M. Benhamou, M. Hamouni, A. J. Lozano Guerrero, Modeling of Electromagnetic Shielding Properties of Conductive sheet using the

matrix formalism, $4^{\text {th }}$ International Conference on Electrical Engineering (ICEE), pp., 1-4, 13-15 December, 2015

[2] R. P. Clayton, Introduction to Electromagnetic Compatibility, John Wiley \& Sons, Inc., Hoboken, New Jersey. 2006.

[3] S. M. Benhamou, M. Hamouni, "Theoretical Approach of Electromagnetic Shielding of Multilayer Conductive Sheets", PIER. M, Vol. 41, pp. 167-175, 2015.

[4] K. Naishadham, "Shielding Effectiveness of Conductive Polymers", IEEE Trans. on EMC, Vol. 34, No. 1, pp. 47-50, February 1992.

[5] S. Wei-Li, C. Mao-Sheng, F. Li-Zhen, L. Ming-Ming, L. Yong, W. Chan-Yuan , J. Hong-Fei, "Highly ordered porous carbon/wax composites for effective electromagnetic attenuation and shielding", CARBON, Vol. 77, pp. 130-142, 2014.

[6] M. H. Al-Saleh, "Electromagnetic Interference Shielding Mechanisms of CNT/polymer composites", CARBON, Vol. 47, pp. 1738-1746, 2009.

[7] S. Celozzi, R. Araneo, G. Lovatr, Electromagnetic Shielding, John Wiley \& Sons, Inc., Hoboken, New Jersey.2008.

[8] N.F. Colaneri, L.W. Shacklette "EMI shielding measurements of conductive polymer blends", IEEE Trans. Instrum. Meas., Vol. 41, pp. 291-297, 1992.

[9] C.J. von Klemperer , D. Maharaj, "Composite electromagnetic interference shielding materials for aerospace applications". Compos. Struct. Vol. 91, No. 4, pp. 467-472, December 2009.

[10] M. H. Al-Saleh, "Influence of conductive network structure on the EMI shielding and electrical percolation of carbon nanotube/polymer nanocomposites", Synthetic Met., Vol. 205, pp. 78-84, 2015.

[11] D. Micheli, C. Apollo, R. Pastore, R.B. Morles, S. Laurenzi, M. Marchetti, "Nanostructured composite materials for electromagnetic interference shielding applications", Acta Astronaut., Vol. 69, , pp. 747 757, 2011.

[12] T. Souier, C. Maragliano, M. Stefancich, M. Chiesa, "How to achieve high electrical conductivity in aligned carbon nanotube polymer composites", CARBON, Vol. 64, pp. 150-157, 2013. 